EPJ Web of Conferences 19, 05010 (2012)

DOI: $10.1051 /$ epjconf/20121905010

(C) Owned by the authors, published by EDP Sciences, 2012

\title{
The distribution of the elements in the thin disc from classical Cepheids
}

\author{
B. Lemasle ${ }^{1, a}$, V.V. Kovtyukh ${ }^{2}$, P. François ${ }^{3}$, G. Bono ${ }^{4}$, C.D. Laney ${ }^{5}$, \\ A. Piersimoni ${ }^{6}$, S. Pedicelli ${ }^{4}$, F. Primas ${ }^{7}$ and M. Romaniello ${ }^{7}$ \\ 1 Kapteyn Institute, University of Groningen, PO Box 800, 9700 AV Groningen, \\ The Netherlands \\ 2 Astronomical Observatory, Odessa National University, T.G. Shevchenko Park, \\ 65014 Odessa, Ukraine \\ 3 Observatoire de Paris, GEPI, 61 avenue de l'Observatoire, 75014 Paris, France \\ 4 Università di Roma Tor Vergata, via della Ricerca Scientifica 1, 00133 Roma, Italy \\ 5 South African Astronomical Observatory, PO Box 9, 7935 Observatory, South Africa \\ 6 Istituto Nazionale di Astrofisica, Osservatorio Astronomico di Collurania, via M. Maggini, \\ 64100 Teramo, Italy \\ 7 European Southern Observatory (ESO), Karl Schwarzschild-Strasse 2, 85748 Garching, \\ Germany
}

\begin{abstract}
Classical Cepheids are the most popular primary distance indicator. They allow us to determine the Galactic abundances gradients and to constrain the chemical enrichment of the thin disc. We present here results based on high resolution spectra of 63 Cepheids. They indicate local abundances inhomogeneities in the outer disc $\left(R_{g}>10 \mathrm{kpc}\right)$.
\end{abstract}

\section{CEPHEIDS AND GALACTIC ABUNDANCES GRADIENTS}

Galactic abundances gradients allow to constrain the chemodynamical evolutionary models of galaxies. Among different tracers used to study the gradients (HII regions, O/B type stars, Planetary Nebulae (PNe), Open Clusters), Cepheids present several advantages: they are bright enough to be observed over a large range of Galactocentric distances; their spectra contain a large set of well-defined absorption lines to determine accurate abundances for many elements; thanks to the Period-Luminosity (PL) relations, they are excellent distance indicators. If the existence of Galactic gradients is nowadays widely accepted, the empirical determination of their properties is still debated. For the iron gradient, Cepheids indicate a break in the slope and a steeper gradient toward the inner disk [1, 2]. In the outer disk (beyond $10 \mathrm{kpc}$ ), the gradient is flattening [3,4]. This flattening is also observed in HII regions [5], PNe [6] and Open Clusters [7, 8]. It is well reproduced by chemodynamical models [9]. Some authors [10] also report a second flattening at a lower basement value. A step-like discontinuity around 10-12 kpc has also been proposed, but it seems to be mainly due to selection effects [4]. Indeed, localized abundance inhomogeneities have been reported $[4,11]$, likely due to "recent" supernovae (SNe).

\section{RESULTS}

Our sample contains high resolution spectra of 63 Cepheids. We performed a classical spectroscopic analysis using OSMARCS models [12]. An estimate of temperatures is given by the line depth ratios

\footnotetext{
a e-mail: lemasle@astro.rug.nl
}

This is an Open Access article distributed under the terms of the Creative Commons Attribution-Noncommercial License 3.0, which permits unrestricted use, distribution, and reproduction in any noncommercial medium, provided the original work is properly cited. 

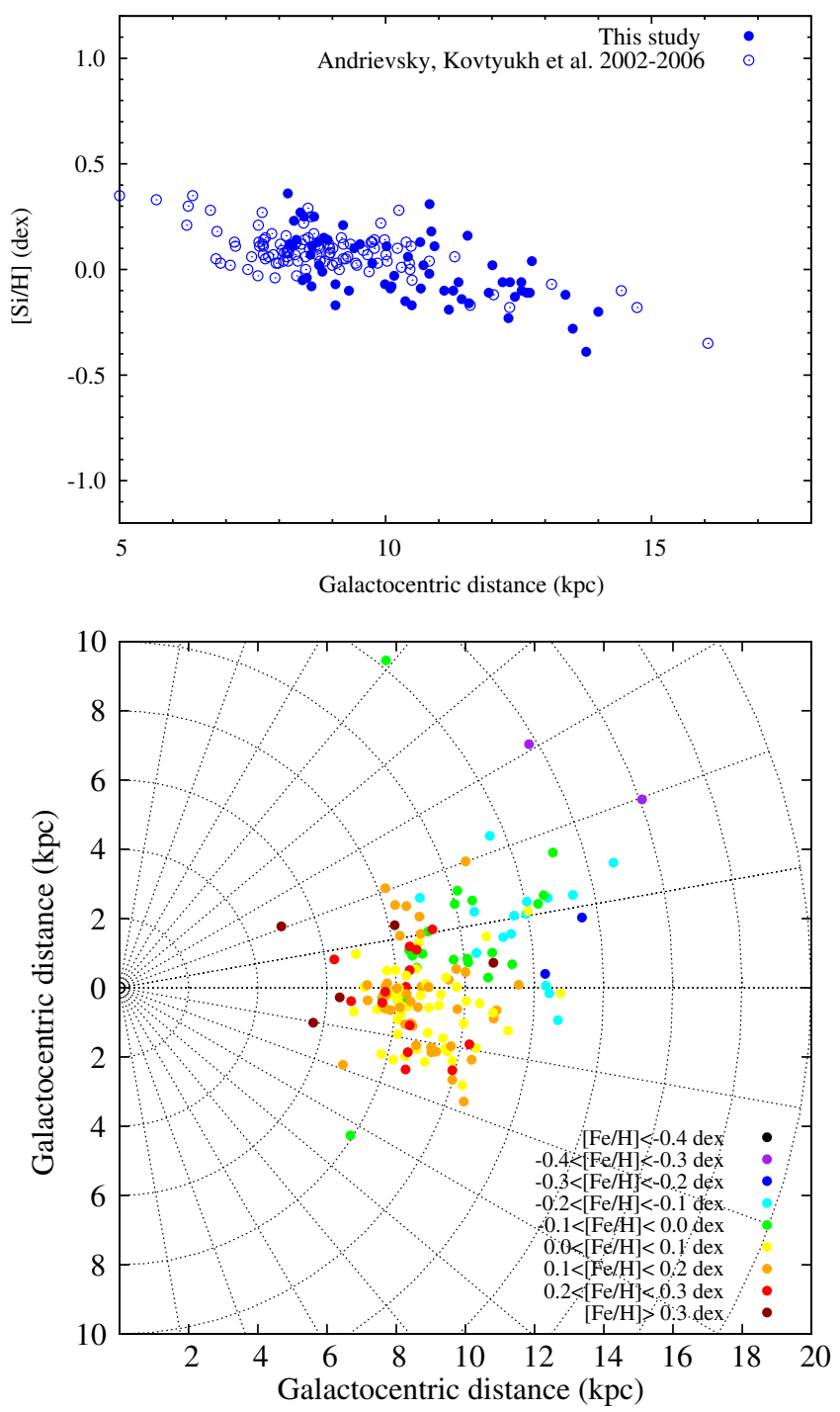

Figure 1. Top: The radial distribution of $[\mathrm{Si} / \mathrm{H}]$. Our data (dots) is shown together with Cepheids whose chemical composition was determined by Andrievsky, Kovtyukh, Luck et al. [1, 3, 11, 18-21] and for which NIR photometry was available (open circles). Bottom: $[\mathrm{Si} / \mathrm{H}]$ distribution in the Galactic disc.

method $[13,14]$. The accuracy of the abundance determination is of 0.12 dex for $[\mathrm{Fe} / \mathrm{H}]$ and of 0.16 dex for the other elements. An independant determination of the abundances using a non-standard method [15] gives results falling within these uncertainties and the differences are often very small. Absolute distances were estimated using near-infrared PL-relations as they are only marginally dependent on the metallicity $[16,17]$ and present a smaller intrinsic dispersion.

We show here results for Si only, but the complete study deals with more than 20 elements. On the top panel of Fig. 1, we show the radial distribution of $[\mathrm{Si} / \mathrm{H}]$. Current data are shown together with Cepheids whose chemical composition was determined by Andrievsky, Kovtyukh, Luck et al. in a series of papers [1, 3, 11, 18-21] and for which near-infrared photometry was available. The gradients for differents elements have different slopes and shapes that reflect the various influences of SNe events and 
AGB stars. The dispersion around the mean locus indicates local inhomogeneities in the Galactic disc. Polar maps (e.g., bottom panel of Fig. 1) show as expected a decrease of all the abundances toward the outer disc. Moreover, the spread is larger in the outer disk, especially when comparing the 10-12 kpc annulus to the one between 8 and $10 \mathrm{kpc}$. This indicates that the interstellar medium is not very well mixed in this region and sensitive to local events such as SNe explosions. Data is up to now too scarce to check if the same outcome applies beyond $12 \mathrm{kpc}$. Due to the strong reddening effects in the thin disc, the excursion in longitude of our sample remains limited.

\section{References}

[1] Andrievsky et al., A\&A 384, (2002b) 140

[2] Pedicelli et al., A\&A 504, (2009) 81

[3] Andrievsky et al., A\&A 413, (2004) 159

[4] Lemasle et al., A\&A 490, (2008) 613

[5] Vilchez \& Esteban, MNRAS 280, (1996) 720

[6] Costa et al., A\&A 423, (2004) 199

[7] Twarog et al., AJ 114, (1997) 2556

[8] Carraro et al., A\&A 476, (2007) 217

[9] Cescutti et al., A\&A 462, (2007) 943

[10] Yong et al., AJ 131, (2006) 2256

[11] Luck et al., AJ 132, (2006) 902

[12] Gustafsson et al., A\&A 486, (2008) 951

[13] Kovtyukh \& Gorlova, A\&A 358, (2000) 587

[14] Kovtyukh, MNRAS 378, (2007) 617

[15] Kovtyukh \& Andrievsky, A\&A 351, (1999) 597

[16] Bono et al., ApJ 512, (1999) 711

[17] Romaniello et al., A\&A 488, (2008) 731

[18] Andrievsky et al., A\&A 381, (2002a) 32

[19] Andrievsky et al., A\&A 392, (2002c) 491

[20] Luck et al., A\&A 401, (2003) 939

[21] Kovtyukh et al., AJ 129, (2005a) 433 